\title{
THE FOLDED RIBBON THEOREM FOR REGULAR CLOSED CURVES IN THE PLANE
}

\author{
BY GEORGE K. FRANCIS ${ }^{1}$
}

Communicated by Lamberto Cesari, November 9, 1967

Let $S$ be the oriented circle with base point, $E$ the oriented Euclidian plane, and $V$ the positively oriented two frames in $E$. Let $L$ be the space of $C^{1}$-regular immersions $g: S \rightarrow E$ with continuous right transverse field $\hat{g}$. For $g \in L$, set $\partial g=\left(g, \hat{g}, g^{\prime}\right): S \rightarrow E \times V$. A positive monotone regular homotopy (=monotopy) from loop $g_{-1}$ to $g_{+1}$ is a $C^{\mathbf{1}_{-}}$ regular homotopy $G:[-1,+1] \times S \rightarrow E$ with positive Jacobian and $\partial G(i, x)=\partial g_{i}(x), i= \pm 1$, where $\partial G=(G, \partial G / \partial t, \partial G / \partial x)$. A negative monotopy $G$ from $g_{-1}$ to $g_{+1}$ is such that $G^{*}(t, x)=G(-t, x)$ is a positive monotopy from $g_{+1}$ to $g_{-1}$. A monotopy is stronger than a regular homotopy in that the latter requires only that $\partial G / \partial x \neq 0$. The tangent winding number TWN of $g$ in $L$ is the degree of $g^{\prime} /\left|g^{\prime}\right|: S \rightarrow S$. Because degree is a homotopy invariant, regular homotopy preserves the TWN. The converse of this is the Whitney-Graustein Theorem [3]. The TWN actually classifies $L$ in a much stronger fashion.

Theorem. For two regular loops $g_{i}, i= \pm 1$, of like $T W N$, there always is a regular loop $g_{0}$ and two monotopies $H_{i}: g_{i} \sim g_{0}, i= \pm 1$, of like sign equal to sign ( $\left.T W N \pm \frac{1}{2}\right)$.

Note that $\mathrm{TWN}=0$ belongs to both cases. For $\mathrm{TWN}=1$, two concentric circles are monotopic. Not so for two circles whose interiors are disjoint; yet each is monotopic to a circle surrounding them both.

The method of proof is entirely constructive. The normal loops $L_{N}$ have only simple, signed, transverse self-intersections (=nodes). $L_{N}$ is dense and open (=generic) in $L$ under the topology induced by $\|g-h\|=\max |\partial g(x)-\partial h(x)|, x \in S$. (See [3] for details.)

Proposition 1. If $g \in L$ and $\epsilon>0$, there is an $h \in L_{N}$ with $\|g-h\|<\epsilon$ and a monotopy of prescribed sign between them.

The proof of Proposition 1 makes use of a stable condition of "parallelity": min det $\left(g(x)-h(x), t^{\prime}(x)+(1-t) h^{\prime}(x)\right)>0$, over all $x \in S$ and $t \in[0,1]$. The key lemma reads:

LEMma. If $w$ is a continuous, periodic, transverse field along the ordinate in the $(t, x)$-Cartesian plane, then the map $F(t, x)=(t-z(t), x)$

1 This work comprised part of the author's dissertation written under Professor Charles J. Titus at the University of Michigan, 1967. 
$+\frac{1}{2} \int_{x-2}^{x+z} w(s) d s$ is a diffeomorphism of $(0 \leqq t \leqq 1)$ with positive Jacobian, for a suitable bump-function $z(t)$.

Suppose $G$ is a monotopy terminating with loop $g$. If $d$ is a rightframed embedding of a parameter interval $[a, b]$ on which $g$ is one-toone, such that $d[a, b] \cup g[a, b]$ is a Jordan loop, with $\partial d(c)=\partial g(c)$, $c=a, b$, and $\operatorname{sgn}(d, g)$ is given by

$$
\lim (x \rightarrow a+) \operatorname{sgn} \operatorname{det}\left(d^{\prime}(x), g^{\prime}(x)\right)=\lim (x \rightarrow b-) \operatorname{sgn} \operatorname{det}\left(g^{\prime}(x), d^{\prime}(x)\right),
$$

it is called a simple detour of $g$ with $\operatorname{supp}(d)=[a, b]$. It operates on $g$ to give the loop $d g(x)=d(x)$ for $x \in \operatorname{supp}(d),=g(x)$ otherwise.

Proposition 2. If the sign of $G$ is the same as that of $d$, there is a modified monotopy $d G$, terminating in $d g$.

Suppose $d_{1}$ is a simple detour of $g$, and $d_{2}$ is a simple detour of $d_{1} g$. If $\operatorname{supp}\left(d_{1}\right) \cap \operatorname{supp}\left(d_{2}\right)=\varnothing, d_{1}$ is also a detour of $d_{2} g$. Write $\left(d_{1}+d_{2}\right) g=d_{2}\left(d_{1} g\right)$ with $\operatorname{supp}\left(d_{1}+d_{2}\right)=\operatorname{supp}\left(d_{1}\right) \cup \operatorname{supp}\left(d_{2}\right)$. If $\operatorname{supp}\left(d_{i}\right)$ $\supset \operatorname{supp}\left(d_{j}\right), i \neq j$, and both signs are the same, write $\left(d_{2} d_{1}\right) g=d_{2}\left(d_{1} g\right)$, where $\operatorname{supp}\left(d_{2} d_{1}\right)$ is the larger of the two nested intervals. If $d$ is a detour of $g$, set $d^{*}=g \mid \operatorname{supp}(d)$. Then $d^{*}$ is a detour of $d g$, of sign opposite to that of $d$ and with the same support. $\left(d^{*} d\right) g=g$ undetoured.

A formal expression $D$, made up of a finite number of legitimate sums and products of simple detours is a monotone compound detour provided all components have the same sign; otherwise it is a mixed compound.

Proposition 3. Suppose $G_{-1}:[-1,0] \times S \rightarrow E$ is a monotopy $g_{-1} \sim h_{-1} ; D$ is a compound detour with $D h_{-1}=h_{+1} ;$ and $G_{+1}:[0,+1]$ $\times S \rightarrow E$ is a monotopy $h_{+1} \sim g_{+1}$. (1) If $D$ is monotone and $\operatorname{sgn}(D)$ $=\operatorname{sgn}\left(G_{i}\right), i= \pm 1$, then $g_{-1}$ is monotopic to $g_{+1}$ via a modified monotopy $D G_{-1}$ followed by $G_{+}$. (2) If $D$ is mixed and $\operatorname{sgn}\left(G_{-1}\right) \neq \operatorname{sgn}\left(G_{+1}\right)$, then $D=D_{-1}+D_{+1}$, each summand is monotone with $\operatorname{sgn}\left(D_{i}\right)=\operatorname{sgn}\left(G_{i}\right)$, and each $g_{i}$ is monotopic to $g_{0}=D_{-1} h_{-1}=D_{+1}^{*} h_{+1}$ via $D_{-1} G_{-1}$, resp. $D_{+1}^{*} G_{+1}^{*}$.

Associated to $g \in L_{N}$ is a finite, totally ordered set $W(g)$, called the intersection sequence. For convenience, set $W=\{0,1, \cdots, n\}$. It is obtained by setting $N_{0}=g$ (base point), and enumerating the nodes consecutively. Parametrizing $S$ by $[0,2 \pi]$ allows the association to each $k \in W$ also $\left\{x_{k}^{\prime}, x_{k}^{\prime \prime}\right\}=g^{-1}\left(N_{k}\right), 0 \leqq x_{k}^{\prime}<x_{k}^{\prime \prime}<2 \pi$, and the $k$ th subloop $g_{k}=g \mid\left[x_{k}^{\prime}, x_{k}^{\prime \prime}\right]$. For $i<j$ in $W$, there is a trichotomy of binary relations: $i \supset j$ if $x_{i}^{\prime}<x_{j}^{\prime}<x_{j}^{\prime \prime}<x_{i}^{\prime \prime} ; i \mid j$ if $x_{i}^{\prime}<x_{i}^{\prime \prime}<x_{j}^{\prime}<x_{j}^{\prime \prime}$; $i$ if if $x_{i}^{\prime}<x_{j}^{\prime}<x_{i}^{\prime \prime}<x_{j}^{\prime \prime}$. By abuse of language, relations predicated of indices are equally predicated of the corresponding nodes or sub- 
loops. Please see [1], [2] for details of this combinatorial description of $L_{N}$.

If the relation $L$ is void in $W$, the sequence is properly nested. If both $\mid$ and $L$ are void, $W$ is chained. If $N_{0} \in \operatorname{Clos} C_{\infty}(g)$ (=closure of the unbounded component of $E \backslash g(S)$ ), then $g$ is said to start outside. The $k$ th subloop is exterior if $\left\{g\left(x_{k}^{\prime}-s\right), g\left(x_{k}^{\prime \prime}+s\right) \mid s\right.$ sufficiently small positive $\} \subset_{-} C_{\infty}\left(g_{k}\right)$. In $W$ is a canonical properly nested subsequence $E W$, of essential indices, obtained as follows: The initial index 0 is essential. If $q$ is essential, $q_{1}=\min \{j \mid q \supset j\}$ is essential; $q_{k+1}$ $=\min \left\{j \mid q \supset j\right.$ and $\left.q_{k} \mid j\right\}$ is essential, $k=1,2, \cdots, m$. Let $[g / q]$ $=g\left[x_{q}^{\prime}, x_{q(1)}^{\prime}\right] \cup \bigcup_{1 \leq k \leq m-1} g\left[x_{q(k)}^{\prime \prime}, x_{q(k+1)}^{\prime}\right] \cup_{g}\left[x_{q(m)}^{\prime \prime}, x_{q}^{\prime \prime}\right]$. It is a Jordan loop.

Proposition 4. For $g \in L_{N}, E W(g)$ is properly nested. If $W(g)$ is already properly nested, $E W=W$. If $g$ starts outside, every essential subloop is exterior. If $g$ starts outside and $W(g)$ is not already properly nested, there is at least one essential subloop $q$ that links (=there is a $j$ with $q L j$ or $j L q$ ), but no proper subloop of $q$ links. All linking of $q$ occurs on $[\mathrm{g} / \mathrm{q}]$ and $q$ is linked an even number of times.

For a normal loop $g$ starting outside there is a sign computed for each $k$ in $W(g)$ as follows: $\operatorname{sgn}(0)= \pm 1$ according to which $\{g(0) \pm t \hat{g}(0)\}$ sufficiently small $t>0\} \subset C_{\infty}(g) ; \operatorname{sgn}(k)=\operatorname{sgn} \operatorname{det}\left(g^{\prime}\left(x_{k}^{\prime \prime}\right), g^{\prime}\left(x_{k}^{\prime}\right)\right)$. A properly nested sequence $W$ is precanonical if either $W=\{0,1\}$ and $\operatorname{sgn}(0) \neq \operatorname{sgn}(1)$, or if all indices of $W$ have the same sign. A precanonical sequence is canonical if it is also chained. Each tangent winding number class of normal loops has a unique canonical representative $W(g)$.

The various constructions are summed up by

Proposition 5. For $h \in L_{N}$, there is a mixed sum of simple detours $U$, so that $W(U h)=E W(h)$. There is further, a mixed sum $A$, with $\operatorname{supp}(A)$ $\cap \operatorname{supp}(U)=\varnothing$, so that $(U+A) h$ is precanonical. It is canonical for $T W N(h)=0$ or \pm 1 . If $|T W N(h)| \geqq 2$, there is a monotone sum $B$, $\operatorname{sgn}(B)=\operatorname{sgn}(T W N(h)), \quad \operatorname{supp}(B) \cap \operatorname{supp}(U+A)=\varnothing$, so that $(U+A+B) h$ is canonical.

Proposition 6. Let $f_{i}, i= \pm 1$, be canonical of like $T W N$. There are monotone compounds $D_{i}, \operatorname{sgn}\left(D_{i}\right)=\operatorname{sgn}\left(T W N+\frac{1}{2}\right)$, so that $D_{-1} f_{-1}(S)$ $=D_{+1} f_{+1}(S)$.

Assembly of the proof of the theorem. Let $g_{i}, i= \pm 1$ be in $L$, of like $T W N \geqq 0$. (The case $T W N \leqq 0$ is essentially the mirror image.) By Proposition 1 there are positive monotopies $G_{i}: g_{i} \sim h_{i}, h_{i} \in L_{N}$. Apply 
Propositions 5 and 6 to obtain canonical $f_{i}=\left(U_{i}+A_{i}+B_{i}\right) h_{i}$, and (after a suitable reparametrization of all objects indexed by $i=+1$ ) positive compounds $D_{i}$, so that $D_{-1} f_{-1}=D_{+1} f_{+1}$. The constructions were such that (for $\mathrm{TWN} \geqq 2) \operatorname{supp}\left(D_{i}\right) \subset \operatorname{supp}\left(B_{i}\right), \operatorname{sgn}\left(D_{i}\right)=\operatorname{sgn}\left(B_{i}\right)$ $=+1$, and in all cases, $\operatorname{supp}\left(D_{i}\right) \cap \operatorname{supp}\left(U_{i}+A_{i}\right)=\varnothing$. Thus the compounds $U_{i}+A_{i}+D_{i} B_{i}$ are legitimate (read: $B_{i}=$ identity for TWN $=0,1)$. Because $U_{i}+A_{i}$ is a mixed sum, it can be reassociated to read $M_{i}+N_{i}, M_{i}$ monotone positive, $N_{i}$ monotone negative. Further, $\operatorname{supp}\left(N_{i}\right) \subset \operatorname{supp}\left(D_{j}\right), j \neq i$, hence $N_{i}^{*} D_{j}$ is a legitimate positive product. Finally, it is legitimate to write

$$
\left(M_{-1}+N_{+1}^{*} D_{-1} B_{-1}\right) h_{-1}=\left(M_{+1}+N_{-1}^{*} D_{+1} B_{+1}\right) h_{+1}=g_{0} .
$$

The same argument as in Proposition 3 now completes the proof of the main Theorem, where $H_{i}=\left(M_{i}+N_{j}^{*} D_{i} B_{i}\right) G_{i}, i \neq j$.

\section{REFERENCES}

1. Charles J. Titus, $A$ theory of normal curves and some applications, Pacific J. Math. 10 (1960), 1083-1096.

2. - The combinatorial topology of analytic functions on the boundary of a disk, Acta Math. 106 (1961), 45-64.

3. Hassler Whitney, On regular closed curves in the plane, Compositio Math. 4 (1937), 276-284.

UNIVERSITY OF MiCHIGAN 